\title{
Low "quotient" Lp(a) Concentration Mediates Autoimmune Activation and Independently Predicts Cardiometabolic Risk
}

Authors

Affiliations

\author{
A. Onat ${ }^{1}$, N. Çoban ${ }^{4}$, G. Can ${ }^{2}$, M. Yüksel ${ }^{5}$, A. Karagöz ${ }^{6}$, H. Yüksel ${ }^{1}$, E. Ademoğlư ${ }^{3}$, N. Erginel-Ünaltuna ${ }^{4}$
}

Affiliation addresses are listed at the end of the article
Key words

- autoimmune activation

coronary heart disease

- diabetes, type-2

- impaired fasting glucose

- lipoprotein(a)

\section{Abstract}

$\nabla$

Objective: We determined whether U-shaped relationships exist between serum lipoprotein [Lp](a) and cardiometabolic risk.

Methods: In population-based nondiabetic and diabetic middle-aged adults ( $n=1428$ and 241, respectively) who had been genotyped for the $L P A$ rs10455872 $A>G$ polymorphism, we adjusted the $\operatorname{Lp}(\mathrm{a})$ concentration for the effects of genotype and other covariates. Via sex-specific equations we estimated expected $\mathrm{Lp}(\mathrm{a})$ concentration in each participant, and the quotient between observed to expected $\operatorname{Lp}(\mathrm{a})$ values was determined. $\mathrm{Lp}(\mathrm{a})$ and $\mathrm{Lp}(\mathrm{a})$ quotient tertiles served to identify non-linear associations with outcomes.

Results: Incident 81 cases of diabetes and 128 of coronary heart disease (CHD) developed at
5.1 years' follow-up. $\operatorname{Lp}(\mathrm{a})$ concentration was linearly associated with the LPA genotype, gender, total cholesterol, (inversely) fasting insulin, which together with age formed the variables to derive the equations. In logistic regression for incident diabetes, the low $\operatorname{Lp}(\mathrm{a})$ quotient tertile was a predictor (RR 1.95 [95\%CI $1.10 ; 3.47]$ ) alike the low Lp(a) tertile, additively to major confounders. Cox regression models comprising sex, age, LPA genotype, smoking status, systolic pressure and serum HDL-cholesterol disclosed that, compared with the mid-tertile, both low (HR 1.77) and high $\mathrm{Lp}(\mathrm{a})$ quotient tertiles significantly predicted incident CHD, especially in women.

Conclusion: Elevated cardiometabolic risk is conferred by apparently reduced circulating Lp(a) assays supporting the notion that "low" serum $\mathrm{Lp}(\mathrm{a})$, mediating autoimmune activation, is a major determinant of cardiometabolic risk. received $\quad 30.04 .2014$ first decision 12.06 .2014 accepted 23.07.2014

\section{Bibliography}

DOI http://dx.doi.org/ 10.1055/s-0034-1385922

Published online:

October 14, 2014

Exp Clin Endocrinol Diabetes

2015; $123: 11-18$

(c) J. A. Barth Verlag in Georg Thieme Verlag KG Stuttgart · New York ISSN 0947-7349

Correspondence

\section{Prof. Dr. A. Onat}

Nisbetiye cad. 59/24

Etiler 34335

İstanbul

Turkey

Tel.: + 90/212/3516217

Fax: + 90/212/221 1754

alt_onat@yahoo.com.tr
Elevated plasma lipoprotein[Lp](a) is a recognized cardiovascular risk factor [1-3]. It uses atherogenic and prothrombotic pathways via systemic inflammation and endothelial dysfunction. Its glycoprotein apo(a), bound to apoB moiety, is very heterogeneous in size due to a genetically determined kringle IV type 2 copy number variation within the LPA gene on chromosome 6q27 [4]. Risk of coronary heart disease (CHD) is considered to be determined by $\operatorname{Lp}(\mathrm{a})$ level which is mediated by LPA variants. Kringle IV type 2 repeats were found to be inversely related to the $\operatorname{Lp}(\mathrm{a})$ level and the risk of CHD [5-7].

$2 L P A$ variants that were strongly associated with both an increased level of $\mathrm{Lp}(\mathrm{a})$ lipoprotein and an increased risk of coronary disease have been identified [6] which provide support for a causal role of $\operatorname{Lp}(\mathrm{a})$ lipoprotein in CHD. One SNP, rs10455872, has been found to be strongly associated with $\operatorname{Lp}(\mathrm{a})$ concentrations and number of kringle IV-2 repeats, and to exist only among European Caucasians, not in South Asians or Chi- nese [7]. This SNP polymorphism explained a much larger extent of $\operatorname{Lp}(\mathrm{a})$ variation in European Caucasians, 6-fold that of kringle IV-2 repeats [7]. Sex, apo B and LDL-cholesterol levels explained only a small percentage of $\mathrm{Lp}(\mathrm{a})$ concentration variation. Other SNP variants [8] and specific haplotypes have been related to elevated plasma $\mathrm{Lp}(\mathrm{a})$ and CHD risk [9], but these were correlated with 2 rare variants in the apo(a) gene [10].

The question of whether low levels of plasma Lp(a) also might be related to cardiometabolic risk has not received adequate attention. However, it was demonstrated in a meta-analysis that sex- and age-adjusted $\operatorname{Lp}(\mathrm{a})$ concentrations were lower by $11 \%$ in diabetic than in non-diabetic subjects [2]. Moreover, the genetic effect on CHD risk showed significant heterogeneity between the diabetic and the general population [11]. More notably, the lowest quintile of $\operatorname{Lp}(\mathrm{a})$ was found associated with the development of type-2 diabetes in the prospective Women's Health Study [12]. These observations may be explained by a 
mechanism of immune complex formation involving $\operatorname{Lp}(\mathrm{a})$ and interfered assay results due to failure by capture antibodies [13]. We postulated that genotype-specific low or "residual" Lp(a) concentrations might reflect insulin resistance and autoimmune activation. A method of estimating "residual" Lp(a) had been used in the San Antonio Heart study by Rainwater and Haffner in 1998 [14]. They reported in 473 Mexican Americans that sexand age-adjusted residual $\mathrm{Lp}(\mathrm{a})$ concentrations were inversely correlated with fasting insulin and post-load glucose in nondiabetic individuals, independent of $\mathrm{Lp}(\mathrm{a})$ level and lipid measures. We addressed the above stated question with the following study design. We first estimated "expected" Lp(a) concentrations based on LPA genotype and other covariates and determined the quotient $\operatorname{Lp}(\mathrm{a})$ in each participant. Postulating a non-linear relationship between $\operatorname{Lp}(\mathrm{a})$ (or quotient $\operatorname{Lp}(\mathrm{a})$ ) and cardiometabolic risk, we then investigated prospectively the risk of both type- 2 diabetes and CHD by using tertiles of the quotient $\mathrm{Lp}(\mathrm{a})$ as well as assayed $\mathrm{Lp}(\mathrm{a})$ concentrations in multivariable regression models. Findings elucidated several fundamental aspects of mechanisms for the development of cardiometabolic disorders.

\section{Materials and Methods}

$\nabla$

Population sample

The TARF is a prospective survey on the prevalence of cardiac disease and risk factors in adults in Turkey carried out periodically almost biennially since 1990 in 59 scattered communities [15]. It involves a random sample of the Turkish adult population, representatively stratified for sex, age, geographical regions and for rural-urban distribution.

Measurements of serum Lp(a) were made between the surveys 2002 and 2012 (median 2005) of the Turkish Adult Risk Factor Study (TARF)[16] residing in all 7 geographical regions of Turkey. Participants who had genotyping of LPA polymorphism of rs10455872 along with a first $\operatorname{Lp}(\mathrm{a})$ measurement $(n=1669)$ formed the study sample. The TARF study conformed to the principles embodied in the Declaration of Helsinki and was approved by the Istanbul University Ethics Committee. Written informed consent was obtained from all participants. Data were obtained by history of the past years via a questionnaire, physical examination of the cardiovascular system and recording of a resting electrocardiogram (ECG).

\section{Measurement of risk factors}

Waist circumference was measured with the subject standing at the end of gentle expiration at the level midway between the lower rib margin and the iliac crest. Status of cigarette smoking was categorized into current, former and never smokers. Blood pressure (BP) was measured in the seated position on the right arm using an aneroid sphygmomanometer (Erka, Bad Tölz, Germany), after $5 \mathrm{~min}$ of rest, and the mean of 2 recordings was computed.

Sera were obtained from venous blood after an overnight $>11 \mathrm{~h}$ fasting and measurements were made with commercially available kits in a central laboratory in Istanbul. Serum concentrations of total cholesterol, fasting triglycerides, glucose, creatinine and high-density lipoprotein (HDL)-cholesterol (directly without precipitation) were determined using enzymatic kits from Roche Diagnostics (Mannheim, Germany). Concentrations of insulin and sex hormone-binding globulin (SHBG) were meas- ured by chemiluminescence immunoassay utilising Elecysys 1010 immunautoanalyzer. Apolipoprotein (apo) A-I, apo B, and Lp(a) were measured by means of particle-enhanced immunonephelometry with the Behring nephelometer (Behring Diagnostics).

\section{Determination of the LPA rs 10455872 genotypes}

DNA was extracted from peripheral blood leucocytes using a QIAmp $^{\circledR}$ DNA Maxi KIT (Qiagen, Hilden, Germany). The DNA concentrations have been standardized and stored in $8 \times 12$ format at $-20^{\circ} \mathrm{C}$. Unselected 1669 subjects (789 male and 880 female) were examined for LPA rs10455872 genotypes, performed using hydrolysis probes (TaqMan) in Real-Time PCR LightCycler ${ }^{\circledR} 480$ device. Probes were allele specifically labeled with one of the fluorescent dyes FAM (6-carboxy-fluorescein; major allele) and VIC (proprietary dye of Applied Biosystems, Foster City, CA, USA; minor allele) and contained a minor groove binder group and a dark quencher $[17,18]$. The sequences of primers and probes were designed in house using Primer Express software (version 2.0; Applied Biosystems), Primers: ACTCTCAGCTGCCTTCCTCCTT and CATGTTTGTCTTGGGTAACAAGTGA, Probes: FAM-CAGAACCCAATGTGTTTA-MGB and VICAACCCAGTGTGTTTAT-MGB. Primers and probes were synthesized by Applied Biosystems. DNA amplification was set up in 96 well plates (ABGENE Ltd.) Typical $10 \mu$ PCR reaction consisted of $5 \mu$ l LightCycler $^{\circledR} 480$ probe master ready mix (Roche), $0.2 \mu$ l probes and $0.2 \mu$ primers, $3.2 \mu$ ldistilled water. 5 ng genomic DNA was added to PCR mixture. PCR was carried out on LightCycler ${ }^{\circledR} 480$ using the following conditions: $95^{\circ} \mathrm{C}$ for $10 \mathrm{~min}, 95^{\circ} \mathrm{C}$ for $10 \mathrm{~s}, 57^{\circ} \mathrm{C}$ for $30 \mathrm{~s}, 72^{\circ} \mathrm{C} 5 \mathrm{~s}$ ( 45 cycles). Endpoint analysis was assessed using the LightCycler $^{\circledR} 480$ genotyping software.

\section{Definitions}

Individuals with diabetes were diagnosed with criteria of the American Diabetes Association [19], namely when plasma fasting glucose was $\geq 7 \mathrm{mmol} / \mathrm{L}$ ( or 2 -h postprandial glucose $>11.1 \mathrm{mmol} / \mathrm{L}$ ) and/or the current use of diabetes medication. Impaired fasting glucose (IFG) was identified with a fasting level of 5.56$7.0 \mathrm{mmol} / \mathrm{L}$. Homeostasis model assessment (HOMA) of insulin resistance was calculated in participants who had concomitant fasting insulin and glucose measurements at baseline with the equation $=$ fasting insulin $(\mu \mathrm{U} / \mathrm{ml}) \times$ glucose $(\mathrm{mmol} / \mathrm{l}) / 22.5[20]$. Diagnosis of CHD was based on the presence of angina pectoris, of a history of myocardial infarction with or without accompanying Minnesota codes of the ECG [21] or on a history of myocardial revascularization. Typical angina and, in women, age $>45$ years were prerequisite for a diagnosis when angina was isolated. ECG changes of "ischemic type" of greater than minor degree (Codes 1.1-2, 4.1-2, 5.1-2, 7.1) were considered as myocardial infarct sequelae or myocardial ischemia, respectively. CHD death comprised death from heart failure of coronary origin and fatal coronary event.

\section{Determining the "quotient" Lp(a) concentration}

Mean Lp(a) concentration of each genotype in each participant was adjusted separately in sexes for age, fasting insulin and total cholesterol value to derive an expected $\mathrm{Lp}(\mathrm{a})$ value commensurate with findings provided in 0 Table 2 . The observed serum $\mathrm{Lp}(\mathrm{a})$ value was divided to the expected one, "quotient" $\mathrm{Lp}(\mathrm{a})$ concentration, yielding lower (or higher) Lp(a) levels than the observed ones. This definition reflects better (than an inverse one) the proportion of $\mathrm{Lp}(\mathrm{a})$ mass, not picked up by the assay. 


\section{ஜे Бं}

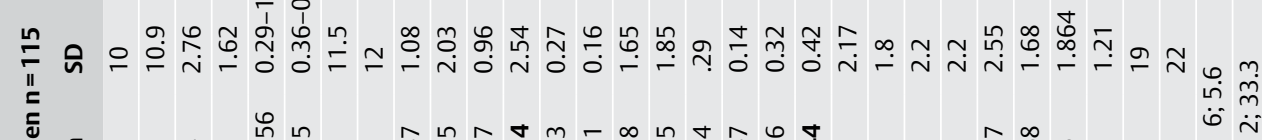

竞

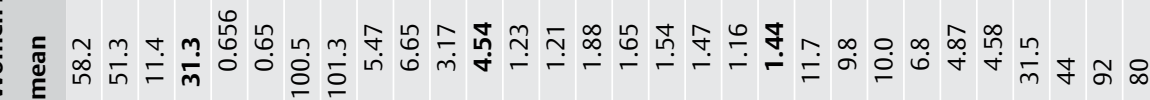

离 至



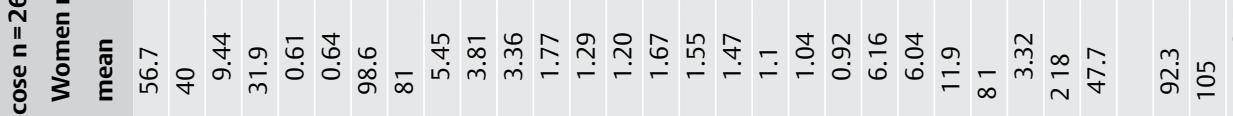
竞 至

它

焉 $\stackrel{\text { i }}{\sim} \stackrel{5}{\sim}$

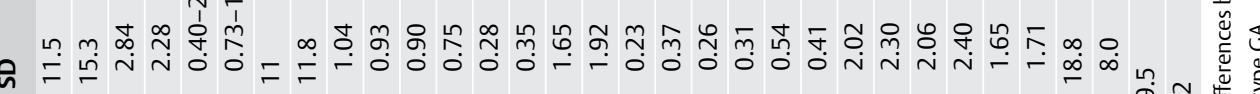



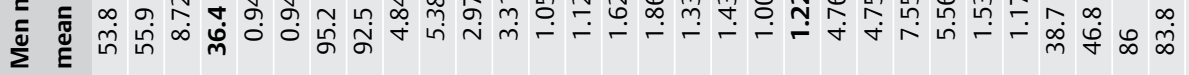

동

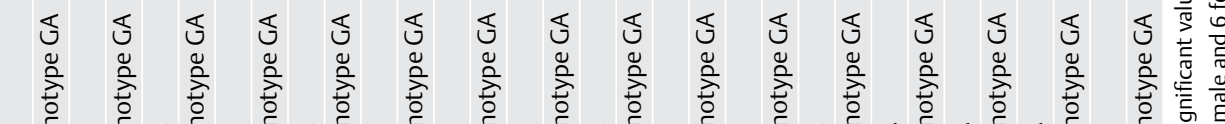

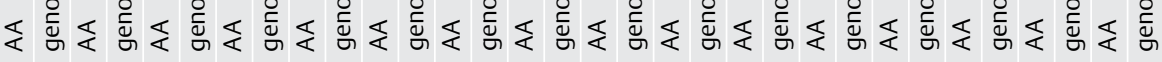


Table 2 Linear regression analysis for $L p(a)$ concentrations in the whole sample, by gender.

\begin{tabular}{|c|c|c|c|c|c|c|c|c|c|}
\hline & B-coeff. & SE & p-value & B-coeff. & SE & p-value & B-coeff. & SE & p-value \\
\hline & \multicolumn{3}{|c|}{ Total, $n=1636$} & \multicolumn{3}{|c|}{ Men, $n=768$} & \multicolumn{3}{|c|}{ Women, $n=868$} \\
\hline female sex & 3.3 & 1.03 & 0.001 & & & & & & \\
\hline LPA GA vs. AA & 28.9 & 3.1 & $<0.001$ & 24.6 & 4.4 & $<0.001$ & 32.3 & 4.0 & $<0.001$ \\
\hline T. cholesterol, $35 \mathrm{mg} / \mathrm{dl}$ & 1.4 & 0.40 & $<0.001$ & 1.54 & 0.56 & 0.006 & 1.11 & 0.52 & 0.04 \\
\hline insulin 2 -fold & -0.54 & 0.36 & 0.04 & -0.75 & 0.40 & 0.062 & -0.34 & 0.46 & 0.45 \\
\hline age, 11 y & 0.1 & 0.55 & 0.7 & 0.59 & 0.64 & 0.35 & -0.29 & 0.66 & 0.066 \\
\hline constant & 8.3 & 3.7 & $<0.001$ & 7.8 & 4.7 & 0.10 & 14.8 & 4.4 & 0.001 \\
\hline
\end{tabular}

The models explained $8 \%$ of $L p(a)$ variation $(p<0.001)$

$2 \%$ of participants had missing insulin values

II log-transformed values

\section{Data analysis}

2-sided t-tests and Pearson's chi-square tests were used to analyze the differences in means and proportions between groups. Differences in the "quotient" $\operatorname{Lp}(\mathrm{a})$ were tested with MannWhitney U test and asymptotic significance. The whole sample was analyzed by stratifying to the presence of baseline diabetic status, gender and LPA genotype. Multiple linear regression analyses were performed with continuous parameters, whereby variables with skewed distribution were log-transformed. The contribution of a significant independent variable as a determinant of $L p(a)$ in a linear regression analysis was calculated by multiplying the related SD value with the $\beta$ coefficient. Relative risk (RR) or hazard ratio (HR) estimates and 95\% confidence intervals $(\mathrm{CI})$ were obtained for "quotient" $\mathrm{Lp}(\mathrm{a})$ and $\mathrm{Lp}(\mathrm{a})$ concentrations with incident elevated HOMA index, diabetes and CHD by use of logistic regression or Cox proportional hazards analyses in models that controlled for potential confounders. Tertiles of quotient $\operatorname{Lp}(\mathrm{a})$ were formed by cutoffs of $0.56 / 0.40$ and 1.54/1.02 constituting the mid-tertile in men and women ( 476,490 , and 475 nondiabetic subjects, respectively), and these were used as dependent variables in predicting cardiometabolic risk. Respective cutoffs for $L p(a)$ were 5.54/7.29 and 16/19.3 mg/ $\mathrm{dl}$ in men and women, respectively. A value of $\mathrm{p}<0.05$ on the 2-tail test was considered statistically significant. Statistical analyses were performed using SPSS-10 for Windows (SPSS Inc., Chicago, Ill).

\section{Results}

$\nabla$

The rs 10455872 genotype GA prevailed in $2.6 \%$ of men, $3.1 \%$ of women. G homozygotes were virtually not represented $(0.045 \%)$ in the TARF cohort. $\mathrm{Lp}(\mathrm{a})$ concentrations corresponding to GA genotype were overall 3.5-fold higher than the AA genotype regardless of gender or diabetic status. Total follow-up of the nondiabetic sample consisted of 7280 person-years (1428 subjects for a mean $5.1 \pm 2.1$ years).

Clinical features of GA -compared with the common- genotype was led by high $\mathrm{Lp}(\mathrm{a})$ concentrations and further by significantly higher apo B in normoglycemic men and LDL-cholesterol in women, along with narrower waist circumference ( $\odot$ Table 1 ). In men with IFG, as distinct from women, higher total and LDLcholesterol as well as lower HOMA index prevailed. (GA genotype seemed to exhibit a lower DM prevalence in males, whereas it interacted with the female gender to tend to be associated with a higher DM prevalence (1.98-fold, $\mathrm{p}=0.094)$ ).

\section{Determinants of $L p(a)$ concentration and derivation of} expected Lp(a) values

Equation for estimating expected $\mathrm{Lp}(\mathrm{a})$ concentration was derived from a multivariable linear regression analysis comprising age, genotype, serum total cholesterol and fasting insulin as independent variables ( $\bullet$ Table 2). LPA genotype was the main determinant followed by female sex, total cholesterol and, inversely, insulin. Sex-specific equations were found as follows.

Male $=7.762+(0.054 *$ age $)+(0.044 *$ TChol $)+(-3.773 *$ LogIns $)+24.62$ if genotype GA

Female $=14.802+\left(-0.027^{*}\right.$ age $)+(0.032 *$ TChol $)+\left(-1.714^{*}\right.$ LogIns) +32.26 if genotype GA

Values in $\triangle$ Table 2 indicate that, apart from the gene variant, female sex independently contributed by $3.3 \mathrm{mg} / \mathrm{dl}, 1-\mathrm{SD}$ increment of total cholesterol by $1.4 \mathrm{mg} / \mathrm{dl}$, and of fasting insulin by $-0.54 \mathrm{mg} / \mathrm{dl}$.

\section{"Quotient" Lp(a), an inverse covariate of HOMA index} Overall "quotient" Lp(a) exhibited in men a median of 0.893 (IQR 0.4 and 2.02) and in women 0.63 (IR 0.292 and 1.306) in the nondiabetic sample $(p=0.001$, significant difference in estimated values across sexes). It was similar among diabetic and nondiabetic subjects. Median (IQR) Lp(a) quotient in nondiabetic homozygotes were significantly lower than in GA genotype carriers only among women ( $\odot$ Table 1, Supplementary Table 5 and Supplementary Fig. 1).

Univariate correlation between log-transformed $\mathrm{Lp}(\mathrm{a})$ and the HOMA index was weakly but uniformly inverse irrespective of gender and diabetic status. It was significant in the whole sample ( $r=-0.065, p=0.023)$. "Quotient" Lp(a) and the HOMA index were uniformly not correlated (Spearman) in normoglycemic subjects; the correlation tended to be insignificantly inverse in individuals with impaired fasting glucose and in diabetic women.

A possible predictive value of $\operatorname{Lp}(\mathrm{a})$ and "quotient" $\mathrm{Lp}(\mathrm{a})$ tertile for future development of HOMA index $>2.5$ was examined in a logistic regression model among nondiabetic participants after exclusion of the sample with baseline HOMA $>2.5$ and those with no follow-up ( $\odot$ Table 3). Though no significant association was observed, the RRs tended to disclose a U-shaped curve in women -more prominent for quotient Lp(a)- and to weakly increase in men with decreasing "quotient" Lp(a) tertiles. 
Table 3 Logistic regression analyses with "quotient" $L p(a)$ and assayed $L p(a)$ for prediction of HOMA index $>2.5$.

\begin{tabular}{|c|c|c|c|c|c|c|}
\hline & \multicolumn{2}{|c|}{ Total } & \multicolumn{2}{|c|}{ Men } & \multicolumn{2}{|c|}{ Women } \\
\hline & $\mathbf{R R}$ & $95 \% \mathrm{Cl}$ & $\mathbf{R R}$ & $95 \% \mathrm{Cl}$ & $\mathbf{R R}$ & $95 \% \mathrm{Cl}$ \\
\hline & \multicolumn{2}{|c|}{$126 / 902 \dagger$} & \multicolumn{2}{|c|}{$54 / 418 \dagger$} & \multicolumn{2}{|c|}{$72 / 484 \dagger$} \\
\hline gender, female & 1.17 & $0.80 ; 1.72$ & & & & \\
\hline age, 11 years & 0.88 & $0.72 ; 1.06$ & 0.82 & $0.61 ; 1.10$ & 0.92 & $0.72 ; 1.17$ \\
\hline Lp(a) quotient low tertile & 1.29 & $0.81 ; 2.06$ & 1.13 & $0.27 ; 2.26$ & 1.42 & $0.75 ; 2.68$ \\
\hline Lp(a) quotient mid-tertile, median $0.89 / 0.63$ & 1 & & 1 & & 1 & \\
\hline Lp(a) quotient high tertile & 1.16 & $0.73 ; 1.84$ & 0.93 & $0.46 ; 1.90$ & 1.32 & $0.71 ; 2.45$ \\
\hline LPA genotype GA vs. AA & 0.99 & $0.33 ; 2.96$ & 0.01 & protective, too few & 1.63 & $0.51 ; 5.26$ \\
\hline \multicolumn{7}{|l|}{ Model 2} \\
\hline Lp(a) low tertile & 1.16 & $0.73 ; 2.34$ & 1.18 & $0.60 ; 2.34$ & 1.13 & $0.60 ; 2.10$ \\
\hline Lp(a) mid-tertile, median $9.4 / 11.9 \mathrm{mg} / \mathrm{dl}$ & 1 & & 1 & & 1 & \\
\hline Lp(a) high tertile & 0.95 & $0.60 ; 1.52$ & 0.91 & $0.45 ; 1.87$ & 0.97 & $0.52 ; 1.80$ \\
\hline
\end{tabular}

†Number of cases/number at risk

Individuals with baseline HOMA> 2.5 and no follow-up were excluded

New insulin resistance developed in 12.9 and $14.9 \%$ of men and women, respectively

Table 4 Logistic regression analyses with Lp(a) "quotient" and assayed Lp(a) tertiles for prediction of incident type-2 diabetes and coronary heart disease.

\begin{tabular}{|c|c|c|c|c|c|c|}
\hline \multirow[b]{3}{*}{ Diabetes } & \multicolumn{2}{|c|}{ Total } & \multicolumn{2}{|c|}{ Men } & \multicolumn{2}{|c|}{ Women } \\
\hline & $\mathbf{R R}$ & $95 \% \mathrm{Cl}$ & $\mathbf{R R}$ & $95 \% \mathrm{Cl}$ & $\mathbf{R R}$ & $95 \% \mathrm{Cl}$ \\
\hline & \multicolumn{2}{|c|}{$81 / 1342 \dagger$} & \multicolumn{2}{|c|}{$43 / 616 \dagger$} & \multicolumn{2}{|c|}{$38 / 726 \dagger$} \\
\hline gender, female & 0.79 & $0.50 ; 1.27$ & & & & \\
\hline age, 11 years & 1.14 & $0.92 ; 1.43$ & 1.20 & $0.89 ; 1.64$ & 1.07 & $0.77 ; 1.49$ \\
\hline waist circumference, $12 \mathrm{~cm}$ & 2.28 & $1.78 ; 2.91$ & 2.28 & $1.60 ; 3.21$ & 2.44 & $1.70 ; 3.50$ \\
\hline IFG vs. normoglycemia & 2.02 & $1.23 ; 3.32$ & 2.44 & $1.24 ; 4.84$ & 1.71 & $0.82 ; 3.58$ \\
\hline Lp(a) quotient low tertile * & 1.95 & $1.10 ; 3.47$ & 1.77 & $0.78 ; 4.02$ & 2.10 & $0.93 ; 4.75$ \\
\hline Lp(a) quotient high tertile * & 1.17 & $0.62 ; 2.18$ & 1.34 & $0.58 ; 3.14$ & 0.87 & $0.34 ; 2.25$ \\
\hline LPA genotype GA vs. AA & 1.66 & $0.37 ; 7.43$ & 0.00 & protective, too few & 7.35 & $1.46 ; 37$ \\
\hline \multicolumn{7}{|l|}{ Model 2} \\
\hline Lp(a) low tertile & 1.98 & $1.13 ; 3.49$ & 1.90 & $0.86 ; 4.20$ & 2.12 & $0.94 ; 4.78$ \\
\hline Lp(a) high tertile & 1.06 & $0.55 ; 2.04$ & 1.28 & $0.53 ; 3.10$ & 0.84 & $0.31 ; 2.25$ \\
\hline Coronary disease & \multicolumn{2}{|c|}{$124 / 1121 \dagger$} & \multicolumn{2}{|c|}{$47 / 505 \dagger$} & \multicolumn{2}{|c|}{$77 / 616 \dagger$} \\
\hline gender, female & 1.44 & $0.91 ; 2.29$ & & & & \\
\hline age, 11 years & 1.43 & $1.18 ; 1.73$ & 0.98 & $0.70 ; 1.41$ & 1.75 & $1.37 ; 2.22$ \\
\hline current smoking & 1.29 & $0.75 ; 2.20$ & 1.19 & $0.58 ; 2.44$ & 1.36 & $0.56 ; 3.32$ \\
\hline former smoking & 0.89 & $0.49 ; 1.64$ & 1.02 & $0.48 ; 2.17$ & 0.72 & $0.18 ; 2.92$ \\
\hline HDL-cholesterol, $12 \mathrm{mg} / \mathrm{dl}$ & 0.78 & $0.64 ; 0.95$ & 0.93 & $0.66 ; 1.31$ & 0.71 & $0.55 ; 0.91$ \\
\hline systolic BP, $24 \mathrm{mmHg}$ & 1.33 & $1.10 ; 1.65$ & 1.57 & $1.10 ; 2.23$ & 1.30 & $1.02 ; 1.65$ \\
\hline Lp(a) quotient low tertile ${ }^{*}$ & 1.77 & $1.10 ; 2.84$ & 1.50 & $0.67 ; 3.36$ & 1.83 & $1.01 ; 3.31$ \\
\hline Lp(a) quotient high tertile * & 1.84 & $1.15 ; 2.94$ & 2.11 & $0.98 ; 4.52$ & 1.74 & $0.94 ; 3.20$ \\
\hline LPA genotype GA vs. AA & 1.07 & $0.34 ; 3.39$ & 1.07 & $0.14 ; 7.86$ & 1.23 & $0.30 ; 5.06$ \\
\hline \multicolumn{7}{|l|}{ Model 2} \\
\hline Lp(a) low tertile & 1.71 & $1.07 ; 2.74$ & 1.59 & $0.72 ; 3.50$ & 1.64 & $0.92 ; 2.95$ \\
\hline Lp(a) high tertile & 1.92 & $1.20 ; 3.08$ & 2.27 & $1.05 ; 4.92$ & 1.70 & $0.93 ; 3.12$ \\
\hline
\end{tabular}

Individuals with baseline diabetes, CHD or no follow-up were excluded

$\dagger$ Number of cases/number at risk. Impaired fasting glucose (IFG) status was uncertain in $3 \%$ of participants

Model 2 comprised all variables in Model 1 beyond $L p(a)$ quotient

${ }^{*}$ Referent was mid-tertile with quotient 0.74-1.26 ( $\left.\mathrm{n}=381\right)$

\section{"Quotient" Lp(a) in prediction of diabetes and CHD}

- Table 4 shows logistic regression models in nondiabetic adults in the prediction of 81 cases of incident diabetes. "Quotient" $\mathrm{Lp}(\mathrm{a})$ tertiles were examined along with sex, age, LPA genotype, waist circumference and IFG. The low $\operatorname{Lp}(\mathrm{a})$ quotient tertile was a significant predictor (RR 1.95 [95\% CI 1.10; 3.47]), additively to waist circumference, IFG and, in women, genotype. The low tertile in women tended to borderline significance (RR 2.10 [0.93; 4.75]), while IFG was not significantly associated. Low Lp(a) concentrations in a similar model proved also inversely associated with incident diabetes in combined gender (RR 1.98 [95\% CI $1.13 ; 3.49])$.

Cox proportional hazard regression analysis disclosed that both low (HR 1.77 [95\% CI 1.10; 2.84]) and high Lp(a) quotient tertiles significantly predicted incident CHD at similar magnitudes in a model comprising sex, age, LPA genotype, smoking status, systolic blood pressure and serum HDL-cholesterol ( $\odot$ Table 4). Low Lp(a) tertile also exhibited significant predictive value. Separate analysis in sexes indicated a differing trend regarding low $\mathrm{Lp}(\mathrm{a})$ quotient tertile: it failed to reach significance in predicting CHD 
in men but did predict in women (RR 1.83 [95\% CI 1.01; 3.31]) (Supplementary Fig. 2).

\section{Discussion \\ $\nabla$}

We confirmed in a representative sample of 1341 middle-aged nondiabetic adults that the LPA SNP rs10455872 genotype was the main determinant of assayed $\operatorname{Lp}(\mathrm{a})$ concentrations. Using a method of estimating expected $\operatorname{Lp}(\mathrm{a})$ concentrations and determining the observed/expected $\operatorname{Lp}(\mathrm{a})$ ("quotient") concentrations, we found prospective evidence that non-genetic determinants affected observed $L p(a)$ levels, rendering "reduced" values. The quotient of $\operatorname{Lp}(\mathrm{a})$ tertiles revealed that, beyond excess $\operatorname{Lp}(\mathrm{a})$ values with respect to CHD risk, the low $\mathrm{Lp}(\mathrm{a})$ quotient tertile conferred -as a novel finding- significantly elevated risk for both CHD and type-2 diabetes, compared with the intermediate tertile. Though low $\mathrm{Lp}(\mathrm{a})$ tertile similarly displayed significant independent predictive value for incident diabetes, it was attenuated regarding CHD risk in women. This was consistent with the notion of $L p(a)$ protein being involved in autoimmune activation, impacting excess cardiometabolic risk while yielding reduced assays [13].

\section{Genotype, ethnicity and sex as determinants of Lp(a) concentrations}

In the cross-sectional multi-ethnic SHARE study, 906 healthy Canadians were analyzed for genomic variation in the LPA locus. Among the LPA SNP genotypes, SNP rs10455872 was found to be present only in European Caucasians and explained in them 28\% of the variation in $\operatorname{Lp}(\mathrm{a})$ concentrations, while Kringle IV-2 copy number -associated also with the genotype- explained an additional $4.2 \%$ of the variation [7]. Sex, LDL-cholesterol and apoB explained only $1.5 \%$, whereas in Chinese and South Asians, no more than a quarter of the variation was explained by the stated variables, due to SNP genotype not being as strongly associated with $\operatorname{Lp}(\mathrm{a})$ concentrations as in Europeans.

Alleles of 2 single nucleotide polymorphisms (SNPs) in the LPA gene, $r s 10455872$ and $r s 3798220$, have been shown to be associated with high plasma levels of $\operatorname{Lp}(\mathrm{a})$ and coronary artery disease (CAD) $[6,8]$. The poorly correlated variants together explained about $36 \%$ of the variance in $\operatorname{Lp}(\mathrm{a})$ levels [6]. Each minor allele of $r s 10455872$ (G; $6.2 \%$ frequency) and $r s 3798220$ (C; $1.4 \%$ frequency) increased $\mathrm{Lp}(\mathrm{a})$ by 2.94 and 3.14 and the risk for CAD by 47 and $68 \%$, respectively [6]. A strong and consistent association between the 2 LPA variants and $\operatorname{Lp}(\mathrm{a})$ levels $[6,22]$ has been demonstrated, and $\mathrm{Lp}(\mathrm{a})$ level adjustments abolished the association between the LPA risk variants and CAD [6], thus supporting the view that the effect on atherosclerotic phenotypes is mediated through $\mathrm{Lp}(\mathrm{a})$ levels. However, nonlinear associations of $\operatorname{Lp}(\mathrm{a})$ with $C A D$ have not been adequately analyzed.

\section{Risk conferring for type 2 diabetes}

Possibly due to a non-significant association between circulating $\mathrm{Lp}(\mathrm{a})$ and diabetes development, studies on this association are scarce. The large prospective Women's Health Study [12] reported that $\mathrm{Lp}(\mathrm{a})$ was inversely associated with the development of type- 2 diabetes, exhibiting roughly $25 \%$ higher relative risk in the bottom quintile compared with the remainder of the sample - a result designated as unexpected. Our findings not only support this association but extend the knowledge in the following ways. First, this association was independent of the most relevant LPA polymorphism. Second, rather than inverse association of assayed $\mathrm{Lp}(\mathrm{a})$ levels, the low tertile of $\operatorname{Lp}(\mathrm{a})$ quotient revealed a nearly 2 -fold relative risk for diabetes, additively to the 2 powerful predictors of waist circumference and IFG. This finding is consistent with the proposed autoimmune activation involving $\operatorname{Lp}(\mathrm{a})$ rendering a fraction escaping from immunoassay. The rare $L P A$ allele additionally imparted significant diabetes risk in women alone.

\section{Risk conferring for CHD}

Our findings indicate that, in addition to excess $\mathrm{Lp}(\mathrm{a})$ concentrations or quotient, the low $\mathrm{Lp}(\mathrm{a})$ quotient predicted CHD incidence, independent of LPA polymorphism and conventional cardiovascular risk factors. The imparted risk was similar in magnitude in the extreme tertiles and was similar or larger than that of the conventional risk factors. The relatively modest CHD hazard ratio estimates of $\operatorname{Lp}(\mathrm{a})$ used as a continuous variable [2] may well be attributed both to the U-shaped risk curve and to using the assayed $\operatorname{Lp}(\mathrm{a})$ concentrations without estimating expected values based on genetic and metabolic determinants of $\operatorname{Lp}(\mathrm{a})$. Our findings confirm and largely extend the information provided by Rainwater and Haffner [14].

Patients with type 2 diabetes differ in metabolic profile from the general population leading to a 3-fold higher cardiovascular risk than non-diabetic subjects [23]. Lp(a) levels are lower in diabetic patients than in non-diabetics $[2,12]$. The genetic determinants for $\operatorname{Lp}(\mathrm{a})$ levels in diabetic patients are little known, as is the issue whether elevated $\operatorname{Lp}(a)$ levels causally affect CVD risk in them. Several small prospective studies among patients with type-2 diabetes have yielded conflicting results [24]. Evidence was found that diabetes status attenuates the relation between $\mathrm{Lp}(\mathrm{a})$ and cardiovascular risk [11]. In prospective analysis of patients with type 2 diabetes of the Nurses' Health Study and the Health Professional Follow-Up Study, no significant association was found between plasma $\mathrm{Lp}(\mathrm{a})$ levels and CVD incidence; a borderline association was found with CVD death. None of the LPA SNPs were associated with CVD risk or mortality either [11]. These results may be explained by our finding that low $\operatorname{Lp}(a)$ quotient representing autoimmune activation significantly predicted diabetes, alike CHD, attenuating indeed, the relation between $\mathrm{Lp}(\mathrm{a})$ and cardiovascular risk.

\section{Role of LPA genotype in the presumable autoimmune processes}

Our analyses indicated that it was the common LPA rs10455872A homozygotes that were associated with significantly lower assayed compared with expected $\mathrm{Lp}(\mathrm{a})$ concentrations, especially in women. This was concomitant with the low $\mathrm{Lp}(\mathrm{a})$ quotient tertile being associated with elevated cardiometabolic risk. The GA genotype exhibited sex interaction with respect to insulin resistance, insofar as men displayed inverse correlation with fasted serum insulin and an inverse trend to predict elevated HOMA index yielding protection against the development of diabetes. Female carriers of the rare allele, on the other hand, had similar insulin levels as the homozygotes, but were associated with excess diabetes risk, additively to the determinants of waist circumference, $\operatorname{Lp}(a)$ quotient and the non-significant IFG. This suggests that, beyond the $\operatorname{Lp}(\mathrm{a})$-activated autoimmunity in A-homozygotes acting as diabetogenic, another factor exists, likely related to the apo(a) moiety (short isoforms with fewer repeats and high $\mathrm{Lp}(\mathrm{a})$ concentrations). 
The hypertriglyceridemic-waist phenotype is a recognized atherogenic metabolic marker $[25,26]$ which we have demonstrated in the TARF to be associated in both sexes with marked excess cardiometabolic risk [27]. This phenotype was independently predicted in women also "paradoxically" by antecedent lower circulating $\mathrm{Lp}(\mathrm{a})$ providing support for an autoimmune mechanism rendering reduced assayed $\mathrm{Lp}(\mathrm{a})$ concentrations [27]. Epidemiological evidence is available that a slow long-term process of enhanced low-grade inflammation that comprises impairment in anti-oxidative, anti-inflammatory and insulinsensitizing properties foremost of apo A-I, apo C-III in HDL and HDL, precedes insulin resistance. The formation of a complex between such protective proteins and inflammation-mediated damage of $\operatorname{Lp}(\mathrm{a})$ and/or other plasma proteins, while contributing to oxidative stress and systemic endothelial dysfunction, simultaneously renders escape of part of $L p(a)$ mass with damaged epitope from the highly specific immunoassay.

$\mathrm{Lp}(\mathrm{a})$ is not the only protein/polypeptide comprised in autoimmune complexes. We have recently published evidence of serum creatinine being likewise involved and predicting future CHD risk in the general population [28] or in people without metabolic disorders [29]. Similarly, low glycated hemoglobin has also been considered to represent an elevated risk state in nondiabetic adults [30].

Implications: Recognition of a notion of low $\mathrm{Lp}(\mathrm{a})$ concentrations as indicator of autoimmune activation with concomitantly elevated cardiometabolic risk, particularly in women, has huge implications regarding public health and prevention since diabetes and CHD are highly prevalent in the middle-age and elderly population. Evidence exists further that underlying autoimmune activation may contribute to chronic kidney disease, inflammatory rheumatic diseases [13] and some other chronic diseases. Such a notion entails renewed epidemiologic research, novel immunoassay methods for polypeptides or proteins with damaged epitopes in proinflammatory state and eventual incorporation of this notion into practice guidelines.

Limitations and strength: This study sample is larger than those of few previous prospective analyses on the combined impact of LPA genotype and Lp(a) concentrations. We relied on single measurements of $\mathrm{LP}(\mathrm{a})$ at baseline, only a minor limitation, because the level of this protein is considered to change little over a lifetime. The estimation of possible $\operatorname{Lp}(\mathrm{a})$ levels is based only to one LPA genotype, but this SNP has been documented to be by far the most relevant determinant in Caucasian populations [7]. Current findings may have lower or limited applicability to populations or population segments less susceptible to impaired glucose tolerance. The current study is, to our knowledge, the first one to prospectively investigate the potential nonlinear associations of assayed or estimated $\operatorname{Lp}(\mathrm{a})$ with the risk of $\mathrm{CHD}$ as well as diabetes, using multivariable adjustments.

\section{Conclusion}

\section{$\checkmark$}

In a middle-aged non-diabetic population-based sample prone to MetS, we estimated circulating $\operatorname{Lp}(\mathrm{a})$ from LPA genotype, sex and minor determinants, and utilized the quotient of observed/ expected serum $\mathrm{Lp}(\mathrm{a})$, apart from assayed $\mathrm{Lp}(\mathrm{a})$ concentrations. An approach using tertiles of $\operatorname{Lp}(\mathrm{a})$ quotient allowed the demon- stration of our postulate, namely, the emergence of effects of a nonlinear association between $\operatorname{Lp}(\mathrm{a})$ quotient and outcome in the follow-up. The high tertile of $\operatorname{Lp}(a)$ quotient confirmed the established predictive value for future development of CHD, but the novel finding was that the low tertile proved an independent determinant not only of type-2 diabetes with a 2-fold relative risk, but also a significant one of incident $\mathrm{CHD}$, additively to conventional cardiovascular risk factors. These findings tended to be more pronounced in women and supported a notion that "low" serum Lp(a) mediating autoimmune activation is a major determinant of either cardiometabolic risk and that apparently reduced circulating $\mathrm{Lp}(\mathrm{a})$ may result from autoimmune-mediated complex formation that precludes the immunoassay to pick up a fraction of the $\operatorname{Lp}(\mathrm{a})$ mass. This observation simultaneously suggests that non-genetic inflammation-related determinants substantially influence the level or quality of $\operatorname{Lp}(\mathrm{a})$.

\section{Acknowledgements}

This work was supported in part by The Research Fund of Istanbul University (project number: 29544). We appreciate the dedicated work of past members in the survey teams.

Conflict of interest: The authors declare that they have no conflict of interest.

\section{Affiliations}

${ }^{1}$ Department of Cardiology, Istanbul University, Istanbul

${ }^{2}$ Department of Public Health, Cerrahpaşa Medical Faculty, Istanbul University, Istanbul

Department of Biochemistry, Medical Faculty, Istanbul University, Istanbul

${ }^{4}$ Department of Genetics, Institute for Experimental Medical Research, Istanbul University, Istanbul

Department of Cardiology, Dicle University, Diyarbakir, Turkey

${ }^{6}$ Department of Medicine, Giresun Educ. Hospital, Giresun, Turkey

\section{References}

1 Marcovina SM, Koschinsky ML, Albers JJ et al. Report of the National Heart, Lung, and Blood Institute workshop on lipoprotein(a) and cardiovascular disease: recent advances and future directions. Clin Chem 2003; 49: 1785-1796

2 Erqou S, Kaptoge S, Perry PL et al. Lipoprotein(a) concentration and the risk of coronary heart disease, stroke, and nonvascular mortality. JAMA 2009; 302: 412-423

3 Nordestgaard BG, Chapman MJ, Ray K et al. European Atherosclerosis Society Consensus Panel. European Atherosclerosis Society Consensus Panel. Lipoprotein(a) as a cardiovascular risk factor: current status. Eur Heart J 2010; 31: 2844-2853

4 Erqou S, Thompson A, Di Angelantonio E et al. Apolipoprotein(a) isoforms and the risk of vascular disease: systematic review of 40 studies involving 58,000 participants. J Am Coll Cardiol 2010; 55: 2160-2167

5 Holmer SR, Hengstenberg C, Kraft HG et al. Associations of the polymorphisms of apolipoprotein(a) gene with lipoprotein(a) levels and myocardial infarction. Circulation 2003; 107: 696-701

6 Clarke R, Peden JF, Hopewell JC et al.PROCARDIS Consortium. Genetic variants associated with $\mathrm{Lp}(\mathrm{a})$ lipoprotein level and coronary disease. N Engl J Med 2009; 381: 2518-2528

7 Lanktree MB, Anand SS, Yusuf S et al. Comprehensive analysis of genomic variation in the LPA locus and its relationship to plasma lipoprotein(a) in South Asians, Chinese, and European Caucasians. Circulation Cardiov Genet 2010; 3: 39-46

8 Luke MM, Kane JP, Liu DM et al. A polymorphism in the protease-like domain of apolipoprotein(a) is associated with severe coronary artery disease. Arterioscler Thromb Vasc Biol 2007; 27: 2030-2036

9 Trégouët DA, König IR, Erdmann J et al. Genome-wide haplotype association study identifies the SLC22A3-LPAL2-LPA gene cluster as a risk locus for coronary artery disease. Nat Genet 2009; 41: 283-285 
10 Koch W, Mueller JC, Schrempf $M$ et al. Two rare variants explain association with acute myocardial infarction in an extended genomic region including the apolipoprotein(A) gene. Ann Human Genet 2013; 77 : 47-55

11 Qi Q Workalemahu T, Zhang $C$ et al. Genetic variants, plasma lipoprotein(a) levels, and risk of cardiovascular morbidity and mortality among two prospective cohorts of type 2 diabetes. Eur Heart J 2012; 33: 325-334

12 Mora S, Kamstrup PR, Rifai $N$ et al. Lipoprotein(a) and risk of type 2 diabetes. Clin Chem 2010; 56: 1252-1260

13 Onat $A$, Can $G$. Enhanced proinflammatory state and autoimmune activation: a breakthrough to understanding chronic diseases. Curr Pharm Des 2014; 20: 575-584

14 Rainwater DL, Haffner SM. Insulin and 2-hour glucose levels are inversely related to $\mathrm{Lp}(\mathrm{a})$ concentrations controlled for LPA genotype. Arterioscler Thromb Vasc Biol 1998; 18: 1335-1341

15 Onat A. Risk factors and cardiovascular disease in Turkey. Atherosclerosis 2001; $156: 1-10$

16 Onat A, Hergenç G, Ozhan H et al. Lipoprotein(a) is associated with coronary heart disease in women independent of metabolic syndrome. Coron Artery Dis 2008; 19: 125-131

17 Kutyavin IV, Afonina IA, Mills A et al. 3'-minor groove binder-DNA probes increase sequence specificity at PCR extension temperatures. Nucleic Acids Res 2000; 28: 655-661

18 De Kok JB, Wiegerinck ETG, Giesendorf BAJ et al. Rapid genotyping of single nucleotide polymorphisms using novel minor groove binding DNA oligonucleotides (MGB probes). Hum Mutat 2002; 19: 554-559

19 Genuth S, Alberti KG, Bennett P et al.Expert Committee on the Diagnosis and Classification of Diabetes Mellitus. Expert Committee on the Diagnosis and Classification of Diabetes Mellitus. Follow-up report on the diagnosis of diabetes mellitus: The Expert Committee on the Diagnosis and Classification of Diabetes Mellitus. Diabetes Care 2003; 26: $3160-3167$
20 Matthews DR, Hosker JP, Rudenski AS et al. Homeostasis model assessment: Insulin resistance and beta-cell function from fasting plasma glucose and insulin concentrations in man. Diabetologia 1985; 28 : 412-419

21 Rose G, Blackburn H, Gillum RF et al. Cardiovascular Survey Methods. 2nd Ed.Geneva, Switzerland: WHO, 1982; 124-127

22 Ronald J, Rajagopalan R, Cerrato $F$ et al. Genetic variation in LPAL2, LPA, and PLG predicts plasma lipoprotein(a) level and carotid artery disease risk. Stroke 2011; 42: 2-9

23 Almdal T, Scharling H, Jensen JS et al. The independent effect of type 2 diabetes mellitus on ischemic heart disease, stroke, and death: a population-based study of 13000 men and women with 20 years of follow-up. Arch Intern Med 2004; 164: 1422-1426

24 Hiraga T, Kobayashi T, Okubo M et al. Prospective study of lipoprotein(a) as a risk factor for atherosclerotic cardiovascular disease in patients with diabetes. Diabetes Care 1995; 18: 241-244

25 Lemieux I, Pascot A, Couillard C et al. Hypertriglyceridemic waist: a marker of atherogenic metabolic triad (hyperinsulinemia; hyperapolipoprotein B; small, dense LDL) in men? Circulation 2000; 102: 179-184

26 Arsenault BJ, Lemieux I, Després JP et al. The hypertriglyceridemicwaist phenotype and the risk of coronary artery disease: results from the EPIC-Norfolk Prospective Population Study. CMAJ 2010; 182: $1427-1432$

27 Onat A, Can G, Örnek E et al. Abdominal obesity with hypertriglyceridemia, lipoprotein(a) and apolipoprotein A-I, determine marked cardiometabolic risk. Eur J Clin Invest 2013; 43: 1129-1139

28 Onat A, Can G, Ademoğlu E et al. Coronary disease risk curve of serum creatinine is linear in Turkish men, U-shaped in women. J Investig Med 2013; 61: 27-33

29 Onat A, Yüksel H, Can G et al. Serum creatinine is associated with coronary disease risk even in the absence of metabolic disorders. Scand J Clin Lab Invest 2013; 73: 569-575

30 Aggarwal V, Schneider ALC, Selvin E. Low hemoglobin A1c in nondiabetic adults. An elevated risk state? Diabetes Care 2012; 35: 20552060 